\title{
Levosimendan: New hope therapy for takotsubo syndrome
}

\author{
Dagmara Hering $^{1,3}$, Milosz Jaguszewski ${ }^{2,3}$ \\ ${ }^{1}$ Dobney Hypertension Centre, School of Medicine and Pharmacology, Royal Perth Hospital Unit, \\ The University of Western Australia, Perth, Australia \\ ${ }^{2}$ First Department of Cardiology, Medical University of Gdansk, Poland \\ ${ }^{3}$ Editorial Office, Cardiology Journal
}

Takotsubo syndrome (TS) is acute usually reversible heart failure (HF) which has gained increasing recognition since first described by Sato et al. in 1990 [1]. Although it has been diagnosed more often over the past decades, the clinical features of TS are complex including multifaceted clinical presentation, often mimicking acute coronary syndrome and symptoms not always preceded by a stressful physical or emotional trigger [2]. In the vast majority, TS affects post-menopausal women ( $\sim 90 \%)$, however $\sim 10 \%$ of males develop TS with a higher risk of more frequent life-threatening complications (i.e. cardiac arrest, ventricular fibrillation, sudden cardiac death etc.). Evidence from numerous case reports and international registries indicates that TS commonly features left ventricle (LV) dysfunction with apical ballooning and contrast retention in the apex and occurs in the absence of culprit atherosclerotic epicardial coronary artery occlusion [3]. However, it may also involve the right ventricle, $L V$ with many anatomical variants and can present with co-existing coronary artery disease (CAD) [2, 4]. Generally, TS is regarded as a reasonably benign condition with a good long-term prognosis and diagnostic criteria nearly resolved $>4$ weeks [5]. However, in the acute phase TS can result in HF, cardiogenic shock, arrhythmia, mitral regurgitation, ventricle thrombus, $\mathrm{LV}$ outflow tract obstruction and cardiac rupture with up to $4-5 \%$ inhospital mortality, 3-17\% 5-year mortality and with a recurrence rate ranging between $5 \%$ and $22 \%$ over a 5-year period, with the second episode occurring 3 months to 10 years after the first diagnosis $[2,3,6]$. While this clinical experience has advanced our knowledge, leading to a better understanding of clinical features and anatomical variants which makes TS more complex and multifactorial than originally thought with the underlying pathophysiology still unclear. Therefore, therapeutic management for TS is still under debate and remains empirical with no evidence from randomized clinical trials available to date. Moreover, the use of catecholamine infusion is controversial and should be rather avoided in compromised TS, likely inducing or worsening ventricle dysfunction. In this context, levosimendan may become a new player in the field of TS therapy. Indeed, levosimendan currently indicated for the treatment of acute decompensated HF appears to be a promising therapeutic approach in TS patients [7]. Levosimendan exerts both inotropic and vasodilatatory effects via increasing cardiac contractility mediated by calcium sensitization of troponin $\mathrm{C}$ and producing vasodilation through opening adenosine triphosphate (ATP)-sensitive potassium channels in vascular smooth muscle cells. Aside from its clinical indication in treating acute decompensated HF, benefits of levosimendan have been demonstrated in other clinical conditions including cardiogenic or septic shock, cardiac surgery and advanced chronic HF [8-10]. First evidence for the use of levosimendan comes from 4 case reports who developed TS-related cardiogenic shock [11-13] or sepsisassociated TS [14]. Further support demonstrating the safety, feasibility and efficacy of levosimendan has been reported in a first prospective pilot study which included 13 consecutive patients with TS and severe ventricle dysfunction [15]. A continuous infusion of levosimendan at a dose of $0.1 \mu \mathrm{g} / \mathrm{kg} / \mathrm{min}$ within the first 24 -h of hospital admission improved

Address for correspondence: Dagmara Hering, MD, PhD, Dobney Hypertension Centre, School of Medicine and

Pharmacology, Royal Perth Hospital Unit, The University of Western Australia, Level 3 MRF Building, Rear 50 Murray Street, Perth WA 6000 MDBP: M570, Australia, tel: +61 89224 0316, fax: +61 89224 0374, e-mail: dagmara.hering@uwa.edu.au 
LV ejection fraction $(28 \pm 5 \%$ at admission vs. $51 \pm 8 \%$ at discharge, $\mathrm{p}<0.001)$. In general, levosimendan was well-tolerated however there was 1 case of sinus tachycardia and a case of torsade de point without inducing ventricular tachycardia. Average hospital stay was $10 \pm 4$ days and all patients were followed-up on average over 441 days.

In this issue of 'Cardiology Journal' Yaman et al. [16] provide first evidence for the effectiveness of a loading dose of levosimendan administered in the acute phase in TS patients with LV dysfunction. An infusion of levosimendan at a rate of $0.1 \mu \mathrm{g} /$ $/ \mathrm{kg} / \mathrm{min}$ with its loading dose of $10 \mu \mathrm{g} / \mathrm{kg}$ added to standard-drug therapy (i.e. ACE/ARB, beta-blockers) reduced recovery time with $\mathrm{LV}$ ejection fraction returning to $>50 \%$, the time for normalization of troponin values and hospitalization in TS patients $(n=17)$ when compared to those TS patients $(n=25)$ who did not receive levosimendan within the first $24 \mathrm{~h}$ following hospital admission. All patients at admission demonstrated the absence of CAD in angiogram and demonstrated acute LV dysfunction triggered by preceding physical or emotional stress. While there were no electrocardiographic changes at follow-up between both groups, notably the recovery period associated with levosimendan therapy was reduced.

Although this study is retrospective and nonrandomized which could be viewed as a limitation, authors deserve credit for conducting this investigation further aimed at determining the role of a loading dose of levosimendan in the treatment of TS. The patient follow-up was inadequate to assess prognosis and recurrence rate over the longer term following levosimendan therapy which hopefully further multicentre randomized clinical trials will address. Given the uncertainties regarding the TS pathophysiology there is a need to continue with further research to unravel mechanisms underlying this condition which is integral to establish a treatment for TS.

Conflict of interest: None declared

\section{References}

1. Sato H, Tateishi H, Uchida T. Takotsubo-type cardiomyopathy due to multivessel spasm. In: Kodama K, Haze K, Hon M eds. Clinical aspect of myocardial injury: From ischemia to heart failure. Kagakuhyouronsha, Tokyo, Japan 1990: 56-64.

2. Lyon AR, Bossone E, Schneider B et al. Current state of knowledge on Takotsubo syndrome: A Position Statement from the Taskforce on Takotsubo Syndrome of the Heart Failure Association of the European Society of Cardiology. Eur J Heart Fail, 2016; 18: 8-27.

3. Templin C, Ghadri JR, Diekmann J et al. Clinical features and outcomes of takotsubo (stress) cardiomyopathy. New Engl J Med, 2015; 373: 929-938.

4. Ghadri JR, Ruschitzka F, Luscher TF, Templin C. Takotsubo cardiomyopathy: Still much more to learn. Heart, 2014; 100: 1804-1812.

5. Eitel I, von Knobelsdorff-Brenkenhoff F, Bernhardt P et al. Clinical characteristics and cardiovascular magnetic resonance findings in stress (takotsubo) cardiomyopathy. JAMA, 2011; 306: 277-286.

6. Jaguszewski M, Fijalkowski M, Nowak R et al. Ventricular rupture in Takotsubo cardiomyopathy. Eur Heart J, 2012; 33: 1027.

7. Nieminen MS, Fruhwald S, Heunks LM et al. Levosimendan: current data, clinical use and future development. Heart Lung Vessel, 2013; 5: 227-245.

8. Pierrakos C, Velissaris D, Franchi F, Muzzi L, Karanikolas M, Scolletta S: Levosimendan in critical illness: a literature review. J Clin Med Res, 2014; 6: 75-85.

9. Nieminen MS, Buerke M, Cohen-Solal A et al. The role of levosimendan in acute heart failure complicating acute coronary syndrome: A review and expert consensus opinion. Int J Cardiol, 2016; 218: 150-157.

10. Farmakis D, Alvarez J, Gal TB et al. Levosimendan beyond inotropy and acute heart failure: Evidence of pleiotropic effects on the heart and other organs: An expert panel position paper. Int J Cardiol, 2016; 222: 303-312.

11. Padayachee L. Levosimendan: The inotrope of choice in cardiogenic shock secondary to takotsubo cardiomyopathy? Heart Lung Circ, 2007; 16 (suppl. 3): S65-S70.

12. De Santis V, Vitale D, Tritapepe L, Greco C, Pietropaoli P. Use of levosimendan for cardiogenic shock in a patient with the apical ballooning syndrome. Ann Int Med, 2008; 149: 365-367.

13. Antonini M, Stazi GV, Cirasa MT, Garotto G, Frustaci A. Efficacy of levosimendan in Takotsubo-related cardiogenic shock. Acta Anaesthesiol Scand, 2010; 54: 119-120.

14. Karvouniaris M, Papanikolaou J, Makris D, Zakynthinos E. Sepsis-associated takotsubo cardiomyopathy can be reversed with levosimendan. Am J Emerg Med, 2012; 30: 832.e5-7.

15. Santoro F, Ieva R, Ferraretti A et al. Safety and feasibility of levosimendan administration in takotsubo cardiomyopathy: A case series. Cardiovasc Ther, 2013; 31: e133-e137.

16. Yaman M, Arslan U, Kaya A et al. Levosimendan accelerates recovery in patients with takotsubo cardiomyopathy. Cardiol J, 2016; 23: 610-615. 